\title{
BEZOAR DE MADEIRA EM TRATO GASTROINTESTINAL: RELATO DE CASO E REVISÃO DA LITERATURA
}

\author{
Wood bezoar in the gastrointestinal tract: case report and literature review \\ Jair Gimenez MARRA, Ary Leite de Campos SOBRINHO, Kreisky de Los Santos REBAZA, \\ Josenalda Félix Flores ARATANI, Eloísa Almeida CURVO
}

ABCDDV/577

\begin{abstract}
Marra JG, Sobrinho ALC, Rebaza KLS, Aratani JFF, Curvo EA. Bezoar de madeira em trato gastrointestinal: relato de caso e revisão da literatura. ABCD Arq Bras Cir Dig 2007;20(4):293-6

RESUMO - Introdução - Bezoares são concreções de material animal, vegetal ou mineral retidas do tubo digestivo, sendo mais comuns fitobezoares e tricobezoares. Eles são causadores de várias doenças digestivas, incluindo obstruções, intussuscepções, sangramentos, perfurações, além de enteropatias, pancreatites, apendicites, icterícia obstrutiva e outras. Objetivo - Fazer revisão de literatura e apresentar um caso de paciente psiquiátrica com volumoso bezoar de madeira. Relato do caso - Mulher de 20 anos portadora de distúrbio neuropsiquiátrico tem história de ingestão de palitos de fósforos e lápis de cor durante quatro anos. Foi admitida com queixa de plenitude, dor abdominal e vômitos. Ao exame apresentava-se hipocorada, emagrecida, abdômen levemente distendido e doloroso. Estudo radiológico revelou numerosa quantidade de corpo estranho impactado na região gástrica atingindo porção proximal do intestino delgado. Realizado tratamento com óleo mineral e soro fisiológico através de gavagem, sendo eliminado grande número de fitobezoares nas fezes. Conclusão - A associação da patologia bezoar com distúrbios psiquiátricos é relevante, podendo apresentar recorrências. Estes pacientes sempre que possível devem ser tratados de modo conservador, sendo a endoscopia o método de escolha, apesar de estar limitada à natureza e a quantidade dos bezoares. A remoção cirúrgica tem na gastrotomia anterior longitudinal e a ordenha cautelosa o procedimento de escolha.

DESCRITORES - Bezoar gastrointestinal. Fitobezoar. Bezoar de madeira.
\end{abstract}

\section{INTRODUÇÃO}

Bezoares são definidos como concreções de material ingerido na luz do trato digestivo, geralmente no estômago, podendo apresentar extensão em continuidade para o intestino delgado ou fragmentação com múltiplas massas detectáveis em qualquer segmento intestinal ${ }^{1}$.

Classificam-se geralmente em fitobezoar, tricobezoar e lactobezoar, de acordo com o material que os compõe.

Os fitobezoares são o subtipo mais comum e compostos por fibras vegetais. Ocorrem geralmente em pacientes adultos submetidos à operação gástrica prévia, distúrbio de acidez ou diminuição da motilidade gástrica. São comuns em idosos que apresentam mastigação deficiente devido à ausência de dentes ou distrofia muscular.

Os tricobezoares são formados por cabelos ou pêlos. Geralmente resultam da ingestão de cabelo humano, bonecas ou pelúcias. O termo síndrome de Rapunzel é comumente usado para designar sua extensão desde o estômago até o cólon. Ele é mais comum em pacientes do sexo feminino. A doença é característica de adolescentes do sexo feminino e mulheres jovens, embora ocorra também com freqüência

Trabalho realizado no Departamento de Clínica Cirúrgica do Hospital Geral Universitário (HGU) da Faculdade de Medicina da Universidade de Cuiabá - UNIC, Cuiabá, MT, Brasil.

Endereço para correspondência: Jair Gimenez Marra, e-mail: marrajair@uol.com.br em crianças de idade escolar. A ocorrência em pré-escolares é referida raramente e existe um único caso em literatura em neonato portador de alopecia congênita, atribuído à ingestão intra-útero de pelos eliminados em decorrência de doença ${ }^{2}$. A ocorrência no sexo masculino é excepcional, sendo referido apenas um caso pediátrico ${ }^{3}$.

Os lactobezoares são formados por coágulos de leite e é doença dos lactentes, descritos basicamente em prematuros.

Outras substâncias, porém, têm sido relatadas em sua composição, tais como medicamentos e até produtos de natureza bizarra como fibras de tapete, lã, plástico, gomalaca, areia, pedras e até cordões de sapato.

O objetivo deste trabalho é fazer revisão de literatura e apresentar um caso de paciente psiquiátrica com volumoso bezoar de madeira.

\section{RELATO DO CASO}

R.F.L., 20 anos, sexo feminino, portadora de doença hereditária autossômica recessiva associada à desordem psiquiátrica. Foi encaminhada ao Departamento de Clínica Cirúrgica do Hospital Geral Universitário (HGU) da Faculdade de Medicina da Universidade de Cuiabá - UNIC, Cuiabá, MT, Brasil, com história de ingestão de palitos de fósforos e lápis durante quatro anos juntamente à queixa de plenitude, dor abdominal além de vômitos e fezes contendo pedaços de lápis. 
Ao exame apresentava-se hipocorada, emagrecida, discreta taquicardia, abdômen plano, ruídos hidroaéreos presentes, ventre maciço à percussão, flácido e levemente doloroso à palpação percebendo-se massa palpável em fossa ilíaca direita.

Estudo radiológico de abdome total (Figura 1) revelou numerosa quantidade de corpo estranho impactado na região gástrica atingindo porção proximal do intestino delgado. Hemograma apresentava redução dos valores hematimétricos (hemoblobina de 9,3 $\mathrm{g} / \mathrm{dL}$ e hematócrito de $29,9 \%$ ) e discreta leucocitose com $13.900 \mathrm{~mm}^{3}$.

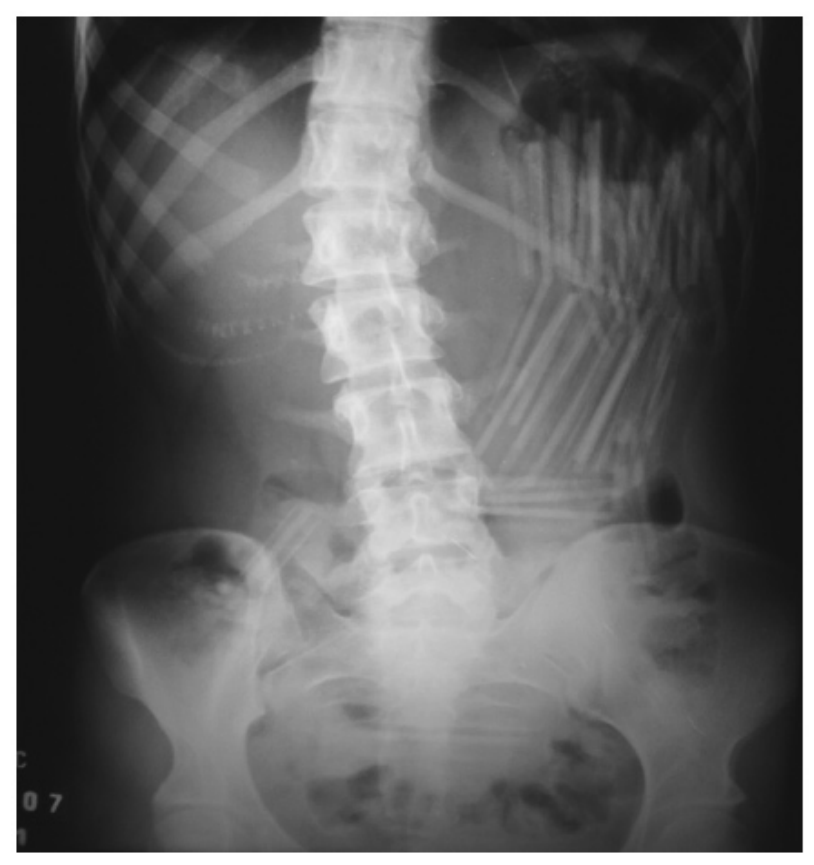

FIGURA 1 - Radiografia de abdome total evidenciando numerosa quantidade de corpo estranho em trato gastrintestinal

Foi iniciado tratamento conservador com administração de óleo mineral, dieta laxativa líquida completa e antiulcerosos além de sulfato ferroso. Evoluiu com eliminação de inúmeros bezoares (lápis) nas fezes. Visando mobilização do conteúdo gástrico remanescente foram realizadas sucessivas gavagens com soro fisiológico $0,9 \%$, que também induziu vômitos com eliminação de parte dos bezoares. Foram também realizadas eletivamente duas explorações endoscópicas no trato digestivo. No primeiro procedimento foi visualizado mais de 15 objetos em sítio gástrico (Figura 2), porém extraídos cautelosamente com alças de polipectomia apenas quatro unidades devido dificuldade técnica e risco de lesão esofágica. Seguiu-se com tratamento conservador já descrito, até imagem radiológica demonstrar ausência de corpo estranho em trato gastrintestinal (Figura 3), para melhor controle foi realizada uma segunda verificação endoscópica (Figura 4) que constatou trato gastrintestinal preservado e presença de apenas um lápis no estômago, sendo ele removido durante essa exploração endoscópica.

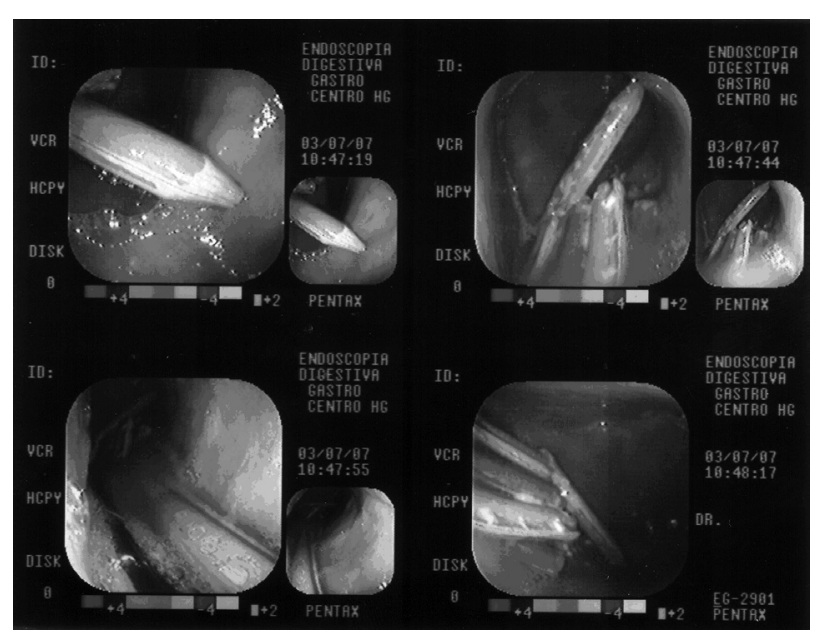

FIGURA 2 - Endoscopia digestiva evidenciando lápis em trato digestivo

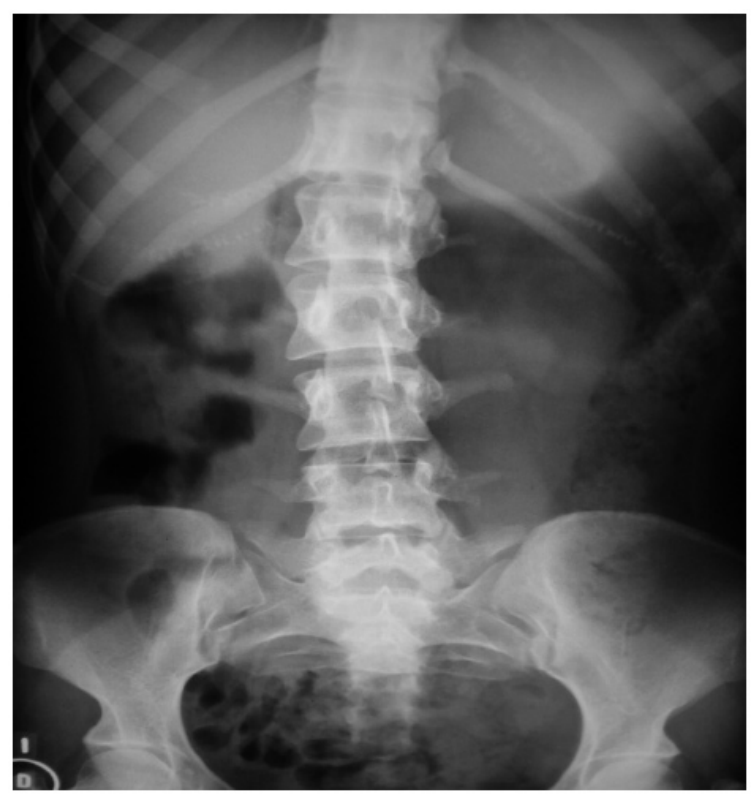

FIGURA 3 - Radiografia de abdome total demonstrando ausência de corpo estranho em trato digestivo

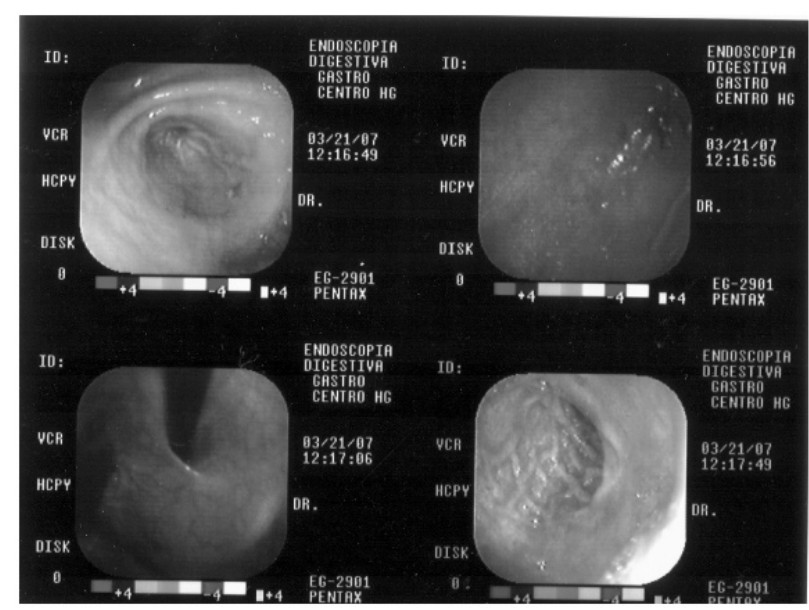

FIGURA 4 - Endoscopia digestiva evidenciando trato digestivo preservado e ausência de corpo estranho. 
No $31^{\circ}$ dia de internação, após remoção de todo bezoar do trato digestivo, que totalizou 119 lápis e três pequenos pedaços de borracha (Figura 5), foi concedido alta hospitalar para a paciente com orientações e encaminhamento ao serviço psiquiátrico.

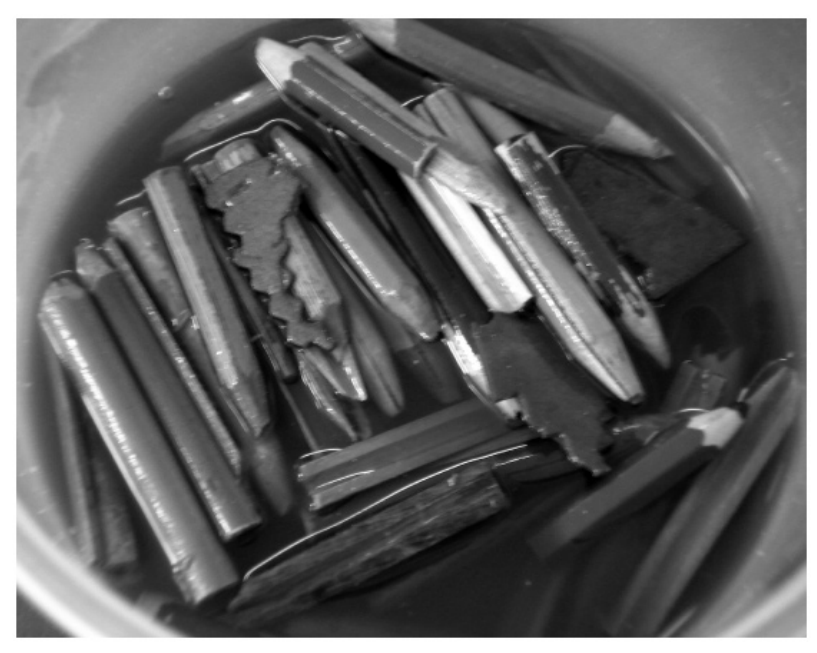

FIGURA 5 - Bezoares retirados do trato gastrintestinal

\section{DISCUSSÃO E REVISÃO DE LITERATURA}

Os bezoares têm sido descritos desde o século 12 A.C. O termo de origem árabe "bad-zehr" ou persa "pad-zehr" significa antídoto. Tidos por quem os possuía como de valor inestimável, até meados do século 18 eram listados na farmacopéia de Londres como remédio para todos os males e pragas ${ }^{4}$. À medida que a medicina foi evoluindo, os seus poderes mágicos foram gradativamente se perdendo, tendo hoje, além do seu aspecto bizarro, significado apenas para a clínica cirúrgica.

Baudamant $^{5}$ foi quem primeiro descreveu-os em 1779 em achado ocasional de autópsia, uma paciente portadora de tricobezoar. Coube a Quain, em 1854,5, a descrição do primeiro caso clínico vivo em paciente insano portador de um fitobezoar, originado a partir da ingestão de fibras do coco. A primeira intervenção cirúrgica para a retirada de bezoar, no entanto, foi realizada em 1883 por Schonborn?

Bezoares são considerados raros, mas, provavelmente, a pequena soma de registros na literatura não reflete a ocorrência real do problema. Eles adquiriram importância por serem agentes causadores de complicações e de várias doenças digestivas, incluindo obstruções, intussuscepções, sangramentos, perfurações, além de enteropatias, pancreatites, apendicites, icterícia obstrutiva e outras.

A sintomatologia dos bezoares é inespecífica, insidiosa e gradual e, não raro, intermitente, guardando estreita relação sintomática com a localização. À medida que se avolumam, podem causar anorexia, perda de peso e desconforto abdominal relacionado às refeições. Quando há obstrução, surgem vômitos e episódios de cólicas abdominais como principais sintomas ${ }^{8}$. O exame clínico costuma evidenciar massa palpável, móvel e endurecida na região epigástrica.

O exame ultrassonográfico do abdome e o estudo radiológico contrastado do estômago podem ser úteis no diagnóstico. Não raro, porém, esses exames falham em estabelecer a natureza do bezoar. Wolfson et al. ${ }^{9}$ têm relatado que radiografias simples do abdome - realizadas após a ingestão de bebidas carbonatadas -, demonstram muito mais claramente a natureza dos bezoares do que a ingestão de bário. A endoscopia digestiva alta, todavia, permite a comprovação diagnóstica e o estabelecimento da natureza do bezoar sem qualquer restrição.

Sempre que possível, eles devem ser tratados conservadoramente. O método de escolha eletivo é a endoscopia, que além de diagnóstica pode ser terapêutica. Todavia, a endoscopia acha-se reservada apenas aos pequenos ou médios bezoares, onde ainda é possível a fragmentação da massa e a sua remoção com pinças do tipo fórceps ou com alça de polipectomia.

O tratamento cirúrgico está reservado, todavia, aos casos de bezoares muito volumosos, ou para os pacientes portadores de complicações. A operação de escolha tem sido a gastrotomia anterior com remoção do bezoar, por vezes associada à enterotomia.

O objetivo final do tratamento, porém, deve ser a abordagem da fitofagia, tricofagia ou outra, bem como a prevenção da recorrência. Embora ainda discutível se existe nitidamente ou não associação entre bezoares e desordens psíquicas, diagnosticadas apenas em $10 \%$ dos $\operatorname{casos}^{10}$, julga-se necessário o encaminhamento de todos os pacientes para serviço de neuropsiquiatria. As recidivas são freqüentes e o tratamento psiquiátrico plenamente justificável.

$\mathrm{Na}$ literatura médica não há relato de bezoar de madeira ou com características semelhantes para nortear qualquer abordagem terapêutica, porém os registros encontrados sobre os bezoares de modo geral, nos remetem a sua grande importância clínica no tocante às complicações e as doenças associadas.

A paciente em questão representou um grande desafio terapêutico, pois apresentava distúrbio psiquiátrico com história crônica de ingestão de corpo estranho e avaliação subjetiva global desfavorável à intervenção cirúrgica imediata. Analisado riscos e benefícios optouse por tratamento conservador sob internação hospitalar e acompanhamento rigoroso para detectar possíveis complicações relacionadas ao bezoar.

O estudo radiológico demonstrou grande importância diagnóstica para o seguimento do tratamento conservador, porém não obteve a mesma acurácia da endoscopia no que se refere ao diagnóstico e tratamento, pois no momento em que a radiografia não evidenciava presença de corpo-estranho em trato gastrintestinal, foi realizada endoscopia de controle, verificando-se ainda a presença de um objeto no estômago que foi retirado endoscopicamente. O estudo endoscópico comprovou que a abordagem terapêutica utilizada foi segura e eficaz, apesar 
da existência de risco de perfuração, broncoaspiração entre outros, não foi necessário realizar intervenção cirúrgica, pois ela poderia trazer mais complicações como a ocorrência de fístulas digestivas de alto débito, visto que o estado nutricional naquele momento era ruim. Deve-se levar em conta ainda a grande chance de recidivas, uma vez que a paciente portava distúrbio neuropsiquiátrico.

\section{CONCLUSÃO}

Os pacientes portadores de bezoares devem, sempre que possível, serem tratados de modo conservador. A endoscopia terapêutica é o método de escolha, apesar de estar limitada à natureza e a quantidade dos bezoares. A remoção cirúrgica tem na gastrotomia anterior longitudinal e a ordenha cautelosa o procedimento de escolha.

Marra JG, Sobrinho ALC, Rebaza KLS, Aratani JFF, Curvo EA. Wood bezoar in the gastrointestinal tract: case report and literature review. ABCD Arq Bras Cir Dig 2007;20(4):

ABSTRACT - Background - Bezoars consist of animal, vegetable or mineral fiber concretions found in the digestive tract, considered to be phytobezoars and trichobezoars the most common forms of presentation. Bezoars cause a number of digestive pathologies including obstructions, intussusceptions, bleeding, perforations as well as enteropathies, pancreatitis, appendicitis, obstructive jaundice and others. Aim - Literature review and case report of a psychiatric patient presenting a large wood bezoar. Case Report - R.F.L, a 20-year-old female, presenting neuropsychiatric disorder and history of ingesting matchsticks and colored pencils for over four years. She was admitted to our service feeling bloated, with abdominal pain and vomiting. Physical examination showed thin patient, pale skin, and slightly distended and painful abdomen. X-ray study showed numerous foreign bodies impacted in gastric region reaching the proximal section of the small intestine. She received tube feeding containing mineral oil and saline solution that resulted in elimination of a large amount of phytobezoars within the excrements. Conclusion - The association of bezoars with psychiatric disorders is relevant and may be recurrent. If possible these patients should be treated conservatively. Endoscopy has been shown to be the technique of choice, although its limitation depends on nature and quantity of bezoars. Surgical removal consists of longitudinal anterior gastrostomy as the preferred surgical procedure.

HEADINGS - Bezoar, gastrointestinal. Phytobezoar. Wood bezoar

\section{REFERÊNCIAS}

1. Jesus Lisieux Eyer de, Novelli Rosa JM. Trichobezoar. Rev. Col. Bras. Cir. [serial on the Internet]. 2005 June [cited 2007 Apr 23]; 32(3): 157-160.

2. Proença E, Carvalho C, Ferreira P, et al. Suboclusión intestinal por tricobezoar congênito. An Pediatr. 2003; 58(2):192-3.

3. Hirugade ST, Talpallikar MC, Deshpande AV, et al. Rapunzel syndrome with a long tail. Indian J Pediatr. 2001; 68(9):895-6.

4. Bockus H. Foreign bodies in the stomach. Gastroenterology Saunders Philadelphia. 1964; 869:874.

5. Baudamant WW. Mémoire sur des cheveux trouvés dans l'estomach et dans l'intestins gréles. J Med Chir Pharm. 1779; 52: 507.

6. Bernstein LH, Gutstein S, Efron G, Wagle A, Graham E. Trichobezoar: an unusual cause of megaloblastic anaemia and hypoproteinaemia in childhood. Am J Dig Dis. 1973; 18: 67.
7. Canavese F, Maiullari E, Costantino S, Rosina F, Gesmundo R, Cortese MG, Bevilacqua A. Tricobezoar gastrico: descrizione di un caso clinico a presentazione anomala. Ped Med Chir. 1994; 16: 289.

8. Dasgupta HK, Chandra SS, Gupta M, Sanwal BL, Bhargawa SC, Vaid RL. Trichobezoar: clinical diagnosis. J Postgrad Med. 1979; 25: 181.

9. Wolfson PJ, Fabius RJ, Leibowitz AN. The Rapunzel Syndrome: an unusual trichobezoar. Am J Gastroenterol. 1987; 82: 365.

10. Lamerton AJ. Trichobezoar: two case reports - A new physical sign. Am J Gastroenterol. 1984; 79: 354.

Conflito de interesse: não há

Fonte financiadora: não há

Recebido para publicação em: 21/05/2007

Aceito para publicação em: 03/09/2007 